\title{
Standoff detection: classification of biological aerosols using laser induced fluorescence (LIF) technique
}

\author{
Anita Hausmann*a, Frank Duschek ${ }^{\mathrm{a}}$, Thomas Fischbach ${ }^{\mathrm{a}}$, Carsten Pargmann ${ }^{\mathrm{a}}$, Valeri Aleksejev ${ }^{\mathrm{b}}$, \\ Larisa Poryvkina $^{b}$, Innokenti Sobolev ${ }^{b}$, Sergey Babichenko ${ }^{b}$, Jürgen Handke ${ }^{a}$ \\ ${ }^{a}$ Institute of Technical Physics, German Aerospace Center (DLR), Langer Grund, D-74239

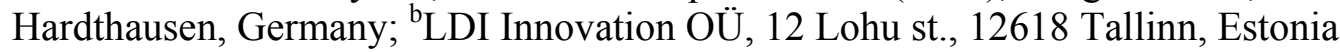

\begin{abstract}
The challenges of detecting hazardous biological materials are manifold: Such material has to be discriminated from other substances in various natural surroundings. The detection sensitivity should be extremely high. As living material may reproduce itself, already one single bacterium may represent a high risk. Of course, identification should be quite fast with a low false alarm rate. Up to now, there is no single technique to solve this problem. Point sensors may collect material and identify it, but the problems of fast identification and especially of appropriate positioning of local collectors are sophisticated. On the other hand, laser based standoff detection may instantaneously provide the information of some accidental spillage of material by detecting the generated thin cloud. LIF technique may classify but hardly identify the substance. A solution can be the use of LIF technique in a first step to collect primary data and - if necessary- followed by utilizing these data for an optimized positioning of point sensors.

We perform studies on an open air laser test range at distances between 20 and $135 \mathrm{~m}$ applying LIF technique to detect and classify aerosols. In order to employ LIF capability, we use a laser source emitting two wavelengths alternatively, 280 and $355 \mathrm{~nm}$, respectively. Moreover, the time dependence of fluorescence spectra is recorded by a gated intensified CCD camera. Signal processing is performed by dedicated software for spectral pattern recognition. The direct comparison of all results leads to a basic classification of the various compounds.
\end{abstract}

Keywords: Standoff detection, CBE, laser induced fluorescence (LIF), hazardous material detection, aerosol

\section{INTRODUCTION}

The release of chemical, biological and explosive (CBE) hazardous materials, whether intentionally or unintentionally, is a very important and recurring topic concerning personal safety and public security. Consistently, the public is shocked by attacks of varying dimensions, for example the disposal of the neurotoxin sarin in a subway in Tokyo 1995 or the transmission of anthrax to American government officials in 2001. Therefore, it is important to find methods, which provide the ability to detect and identify hazardous materials at an early stage without having contact to the danger area, in order to induce the correct counteractions in time to reduce the risk to the public and the emergency services. Especially the detection of biological agents like bacteria is a major challenge. The huge variety of such hazardous substances, their different physical states as well as their different ways of dispersion, contamination and incubation complicate the detection additionally. Besides, aerosols are usually distributed over a broad area, which necessitates a fast detection of the position of the substance source and a survey of the cloud dimensions and movement. ${ }^{[1]}$

Laser based standoff detection methods are distance predicated, discrete, fast, and reliable, which provides promising opportunities and results to early detect and identify various hazardous materials over long distances. The light induced fluorescence (LIF) technique is a comprehensive method, in which the substance itself is excited to emit characteristic radiation. The molecules are raised by laser light to higher energy levels and their subsequent delayed emission is measured. These fluorescence spectra are characteristic for the excited molecules and thus allow a relatively accurate classification. Particularly important is the application under real outdoor conditions in order to consider atmospheric effects. These include, inter alia, the transmission of the laser light, the interference of background radiation, different weather conditions, as well as other substances that may interfere with the observation of hazards. In addition, various natural surroundings like pollen, dust, diesel etc. affect the measurement results and have to be considered in the evaluation process. Furthermore, the detection system must be robust and practiced in busy and public areas, which limits the laser radiation to the eye-safe wavelength range below $400 \mathrm{~nm} \cdot{ }^{[1,2]}$ For these reasons, all LIF measurements 
were studied on a free transmission range with two excitation wavelengths, 280 and $355 \mathrm{~nm}$. A number of different chemical and biological substances were tested for their characteristic fluorescence signals and fluorescence lifetimes. The latter are investigated by a gated iCCD camera, while all data are analyzed with dedicated software for spectral pattern recognition. The direct comparison of all results leads to a first classification of the various compounds. Finally, the substances are aerosolized to simulate a possible realistic propagation of these materials. The results confirm classification capabilities of the method.

\section{EXPERIMENTAL SETUP}

Several substances exhibit broad spectral fluorescence signals upon light excitation, but unfortunately, these broad fluorescence output signals may lead to ambiguous results. In order to improve the discrimination capabilities between signals of different substances and background materials, the laser system operates at two excitation wavelengths, alternatively ${ }^{[3-5]}$ Both, 355 and $280 \mathrm{~nm}$, have a pulse width of about $7 \mathrm{~ns}$, a pulse energy of about $10 \mathrm{~mJ}$ and a repetition rate of $10 \mathrm{~Hz}$. The fluorescence signal is collected by a Newton-type telescope with an optical diameter of $400 \mathrm{~mm}$ and is afterwards analyzed by a spectrograph with a resolution below $1 \mathrm{~nm}$. The detected spectral range covers $300 \mathrm{~nm}$ to $600 \mathrm{~nm}$. Furthermore, the time decay of the fluorescence signal is obtained by using a gated iCCD camera. For the recording of the decay signal, several measurements at slightly shifted gate delay values are necessary. Figure 1 depicts a schematic draft of the aforementioned setup.

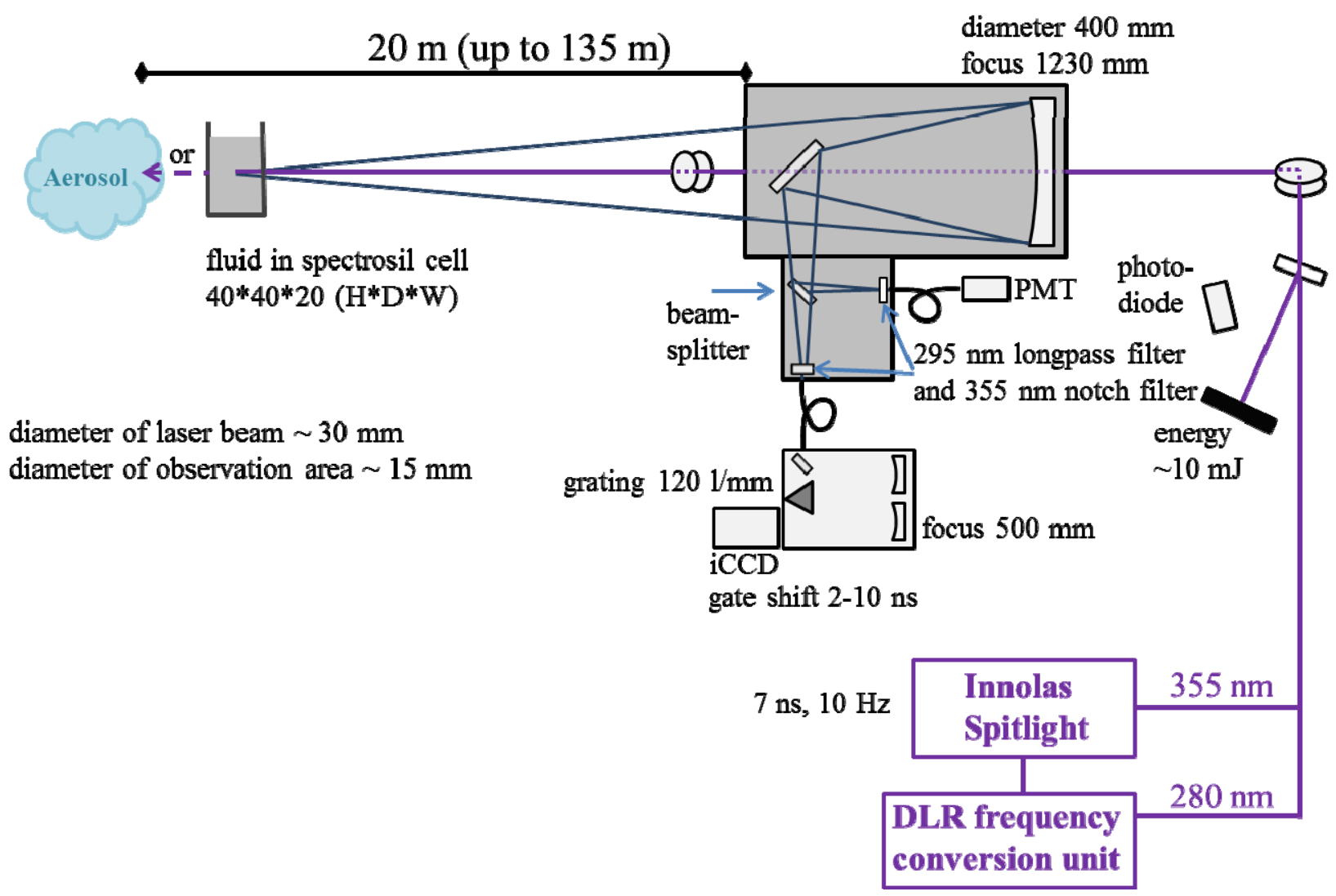

Figure 1. Schematic draft of the LIF measurement setup.

At Lampoldshausen, the German Aerospace Center operates a free transmission range, on which laser based diagnostics, like the laser induced fluorescence (LIF), are tested at distances between 20 and $135 \mathrm{~m}$ under realistic environmental conditions. Figure 2 and 3 show pictures of the LIF measurement composition and the free transmission range, 
respectively. For the reported measurements, the distance of the liquid and/or aerosol samples to the laser and to the telescope is $20 \mathrm{~m}$ (see Figure 1).

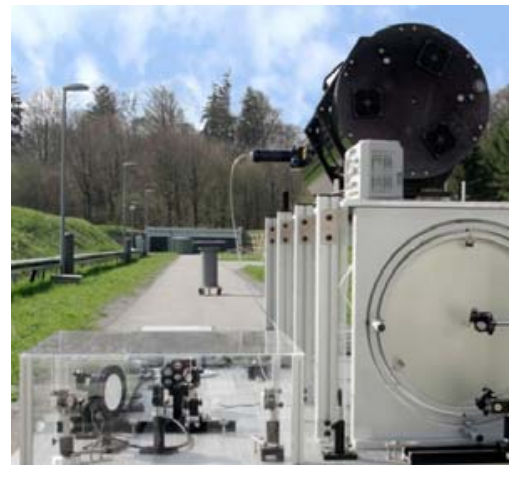

Figure 2. Picture of the LIF measurement composition.

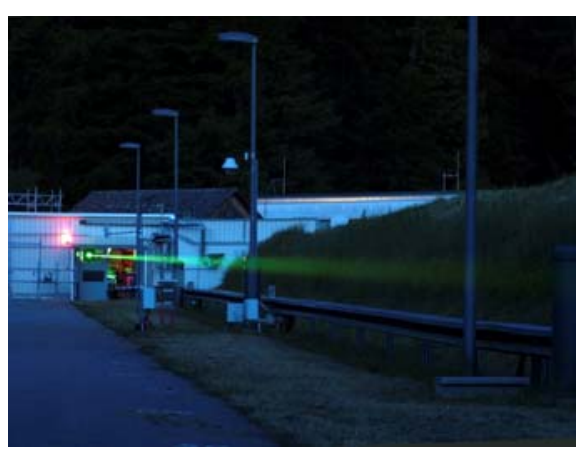

Figure 3. Picture of the free transmission range at night.

\section{INVESTIGATED RESULTS}

In order to demonstrate the capabilities of the setup, different biological substances like fungi, bacteria, vitamins, enzymes, and aromatic amino acids have been measured, as liquid and/or aerosol, respectively. Hydrocarbons and plant materials are exemplary for possible background substances.

To examine both, the concentration dependence as well as the influence of the solvent on the materials, three different approaches per substance are generated. The concentrations are substance dependent and vary between $3.75 \mu \mathrm{g}$ and 3.0 mg per milliliter of deionized water. Because some substances are not completely soluble in water, the corresponding mixtures were stirred for some time (about 20-45 minutes) and then further stirred during the measurement by means of a magnetic stirrer in order to prevent a possible settling of the samples. Previous absorption measurements shed more light on appropriate excitation wavelengths as well as concentrations of the respective sample. Multiple measurements provide information on the reproducibility of the results and the stability against laser irradiation. Delayed fluorescence measurements increase the classification capabilities between different substances by calculating the corresponding fluorescence lifetimes. Afterwards, selected substances are aerosolized to investigate their fluorescence signals under realistic conditions of threats.

In the following, some examples of the obtained fluorescence spectra of different substances are shown (see Figure 4 and 5). The corrected data were obtained by considering the spectral profile of the optical elements and by performing a zero point correction. The application of two excitation wavelengths $(280$ and $355 \mathrm{~nm})$ and the variation of the conditions (liquid : aerosol : solid) leads to additional information. The former helps to discriminate between the different substances, the latter needs to be taken into account due to possible spectral differences and output alternatives.
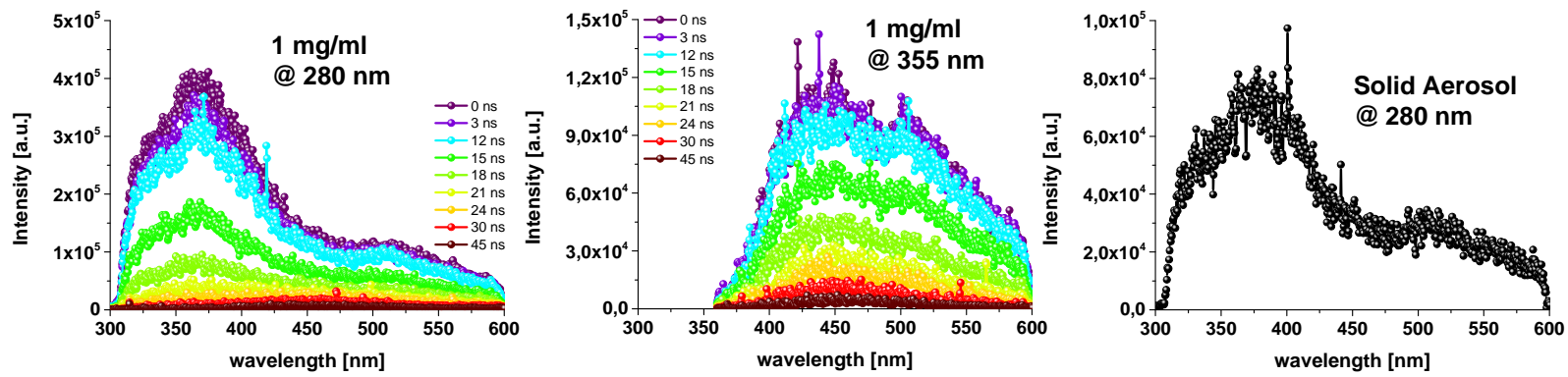

Figure 4. Light induced fluorescence spectra of yeast. Left: $1 \mathrm{mg} / \mathrm{ml}$ deionized water upon $280 \mathrm{~nm}$ excitation with time delays between $0-45 \mathrm{~ns}$ (sum of 100 spectra). Middle: $1 \mathrm{mg} / \mathrm{ml}$ deionized water upon $355 \mathrm{~nm}$ excitation with time delays between $0-45 \mathrm{~ns}$ (sum of 10 spectra). Right: Solid aerosol upon $280 \mathrm{~nm}$ excitation (sum of 100 spectra). 

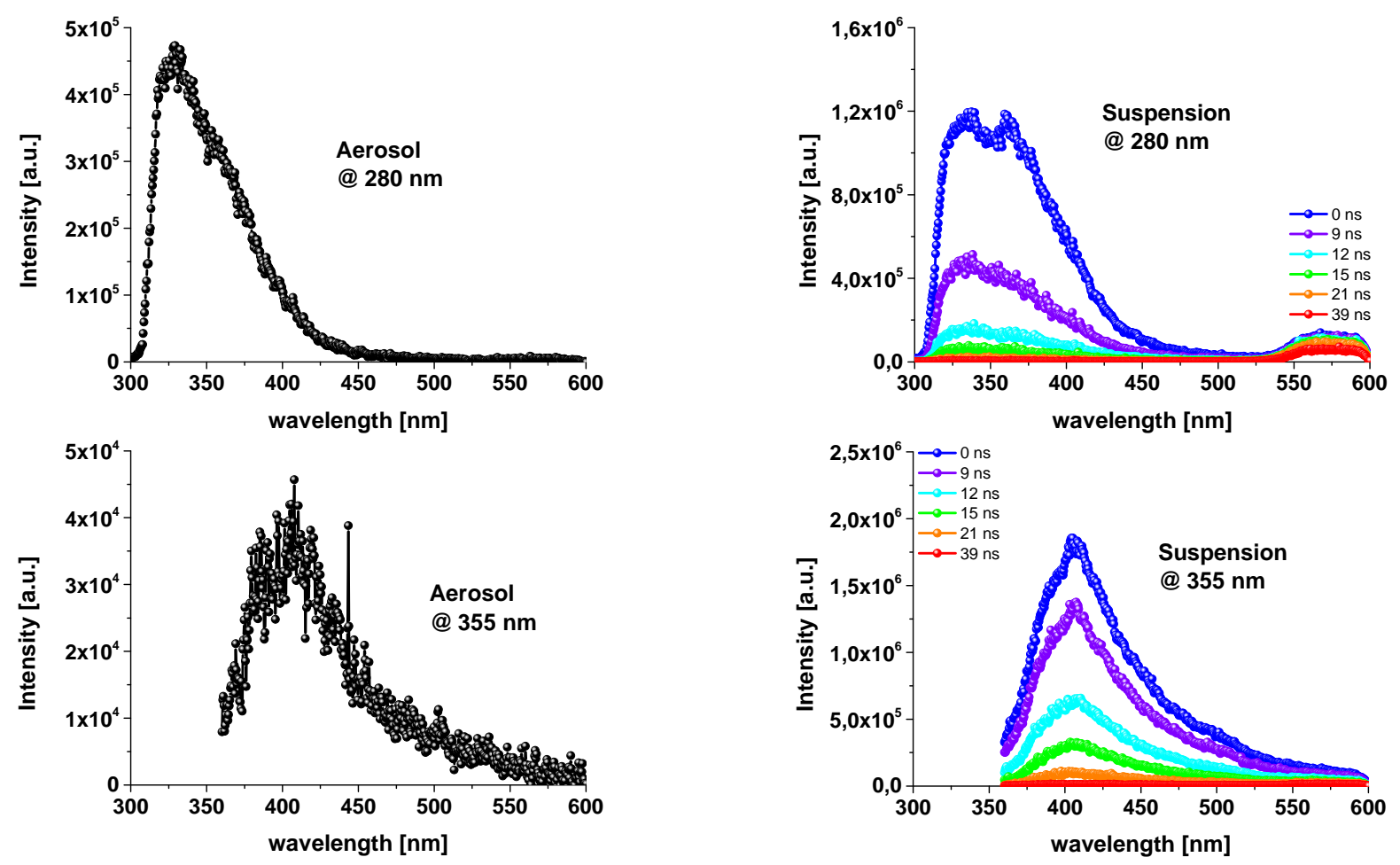

Figure 5. Light induced fluorescence spectra of the amino acid Tryptophan, upper part upon $280 \mathrm{~nm}$ excitation, lower part upon $355 \mathrm{~nm}$ excitation. Left: Aerosol (sum of 100 spectra). Right: Suspension in deionized water with time delays between $0-39 \mathrm{~ns}$ (sum of 10 spectra).

In addition, a distinction of the substances can be carried out by measuring their fluorescence lifetimes. Below, two examples of two different substances are shown, whose fluorescence lifetimes differ drastically (see Figure 6). Both compounds, diesel and dandelion, are excited with $355 \mathrm{~nm}$, but the used gate shift varies from $3 \mathrm{~ns}$ for dandelion to $10 \mathrm{~ns}$ for diesel. When determining the fluorescence lifetimes, the pulse width of the laser is not taken into account, because the calculated values are only used for comparison of the substances.
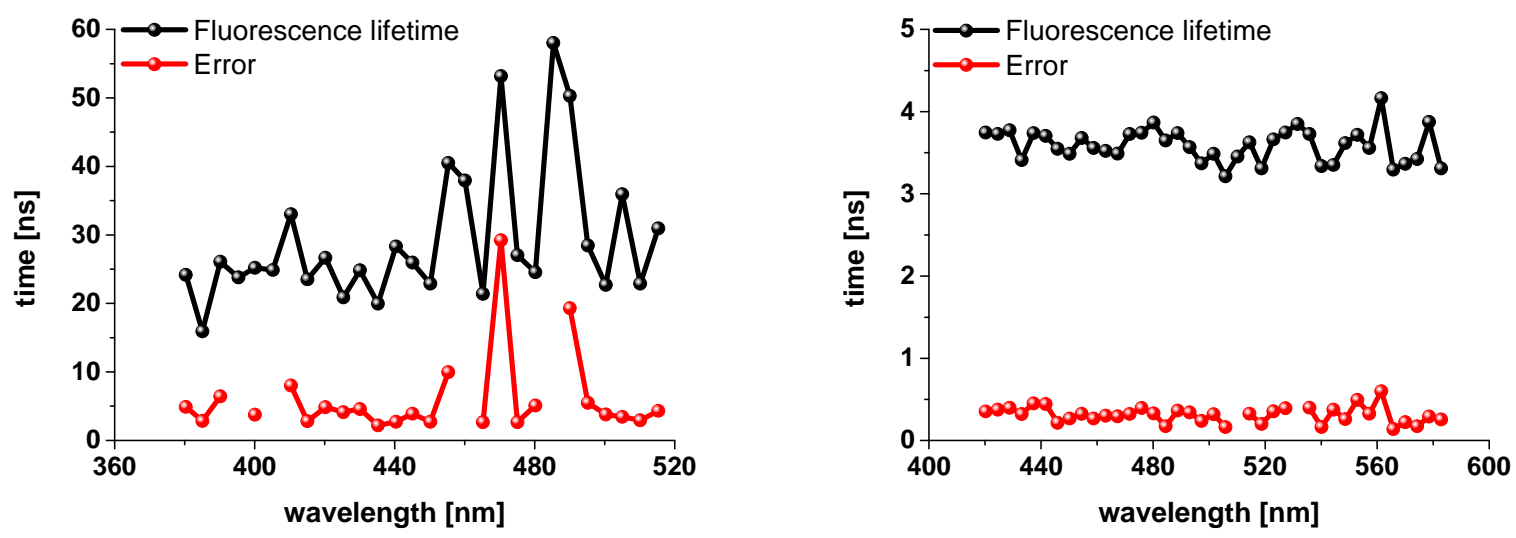

Figure 6. Calculated fluorescence lifetime (black line) and the corresponding error (red line) of diesel (left side) and dandelion (right side) upon $355 \mathrm{~nm}$ excitation. The lifetimes differ in around $30 \mathrm{~ns}$ for diesel and around $3.5 \mathrm{~ns}$ for dandelion. 


\section{SPECTRAL CLASSIFICATION}

Real-time detection of bio-threats should be robust enough for operation in real environment, because hazardous aerosol agents can be present in combination with various natural backgrounds like pollen, dust or exhaust gases, which can significantly affect the measurement results. Application of LIF spectral analysis allows the construction of an automated pattern recognition system for detecting bio-agents. The main objective of the current studies is to develop a system, which is based on an algorithm capable to classify LIF results into four classes, of which the first three constitute the most typical natural background agents and the last one includes live bacteria:

1. chemical aerosols

2. pollen

3. poly-aromatic hydrocarbons (PAHs)

4. live bacteria.

The classification mentioned above is implemented in order to minimize the false positive rate. At the same time, the cost of false negative results can be also very high due to potential exposure of undetected hazardous bio-agents. It provides the challenge of the "cost of error" for the classifier. In order to address such challenge, the hyperspectral LIF (HLIF) data of the measured samples were analysed using a structural feature extraction combined with robust statistical classification. The components of this pattern recognition system are shown in Figure 7.

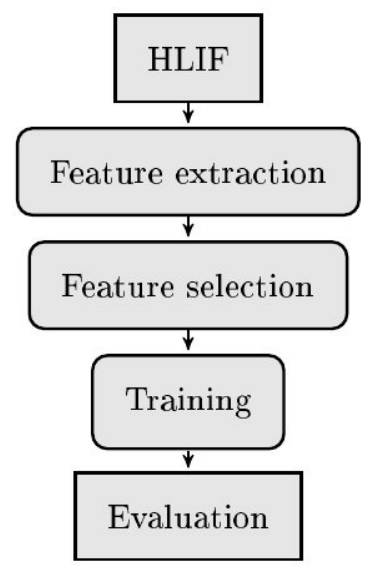

\begin{tabular}{c}
$\begin{array}{c}\text { Measurement of target object } \\
\text { HLIF data in experimental setup }\end{array}$ \\
\hline $\begin{array}{c}\text { Wavelet based extraction } \\
\text { of HLIF structural features }\end{array}$ \\
\hline
\end{tabular}

Selection of class discriminating features using the feature permutation method

\begin{tabular}{c}
\hline $\begin{array}{c}\text { Supervised learning by } \\
\text { bootstrap aggregation on a decision tree }\end{array}$ \\
\hline $\begin{array}{c}\text { The estimation of classificator error } \\
\text { and validation on new dataset }\end{array}$ \\
\hline
\end{tabular}

Figure 7. Components of the bio-threads classification system. The feature extraction module is used to extract the relevant structural information from HLIF data. The feature selection goal is to select the features with most class discriminating capabilities for the proposed class pattern. The training of the statistical classifier on selected features is done using the decision tree bagging method. Test aerosol datasets of chemical and biological agents are used to validate the classifier.

The feature extraction is a critical point in hyperspectral data analysis. The main advantage of hyperspectral data - which details a comprehensive shape of the LIF spectrum - constitutes of a certain drawback for the analysis due to highdimensionality of data space. Various statistical and structural methods are used to reduce the dimensionality of the hyperspectral data. ${ }^{[6,7,8]}$ In particular, it was demonstrated ${ }^{[9]}$ that the wavelet HLIF feature extraction has excellent performance in detection of organic oil pollution in water monitoring application. In this work, a proposed feature extraction according to [9] is executed to each measured LIF spectrum, while these extracted features represent the compressed fluorescence values corresponding to certain spectral ranges in the emission spectra. Thus, each feature is linked with a specific local structure of a HLIF spectrum.

The feature extraction preserves and isolates important and most complete information of the related HLIF spectrum. Additional feature selection is required to find the optimal number of features, which increases the classification performance. To estimate the importance of every extracted feature in class separability, the technique of feature 
permutation was applied. The main idea is to permute the values of each feature across all samples in the dataset and measure the error of class prediction after the permutation.

To create a robust classifier, the method called "bootstrap aggregation" (bagging) on a decision tree was applied. ${ }^{[10]}$ The method generates many replicas of an initial dataset and "grows" the decision trees on these replicas. The training dataset for each decision tree in ensemble is generated randomly by selecting $\mathrm{N}$ samples with replacement from the original data, where $\mathrm{N}$ is the data size. After the training, the answer of this ensemble is created by voting among all decision trees. According to [11], the bagging method leads to the improvements of classification by combining the answers of "weak" classifiers. The unbiased estimator of the true classification error is calculated in the process of training. Therefore, when the size of the experimental dataset is limited, the bagging method delivers additional features as it can be trained without the validation data set. The "Framework for Ensemble Learning" from Matlab Statistics Toolbox was used for implementation of the bagging algorithm.

Data analysis has shown high discrimination capabilities of the proposed recognition system. The plot in Figure 8 represents the clusters of data in scaled coordinates. The scaled coordinates are calculated using the Proximities Matrix Multidimensional Scaling from the tree ensemble, thus they represent the structure of the dataset. The clusters on the plot correspond to the proposed classification pattern.

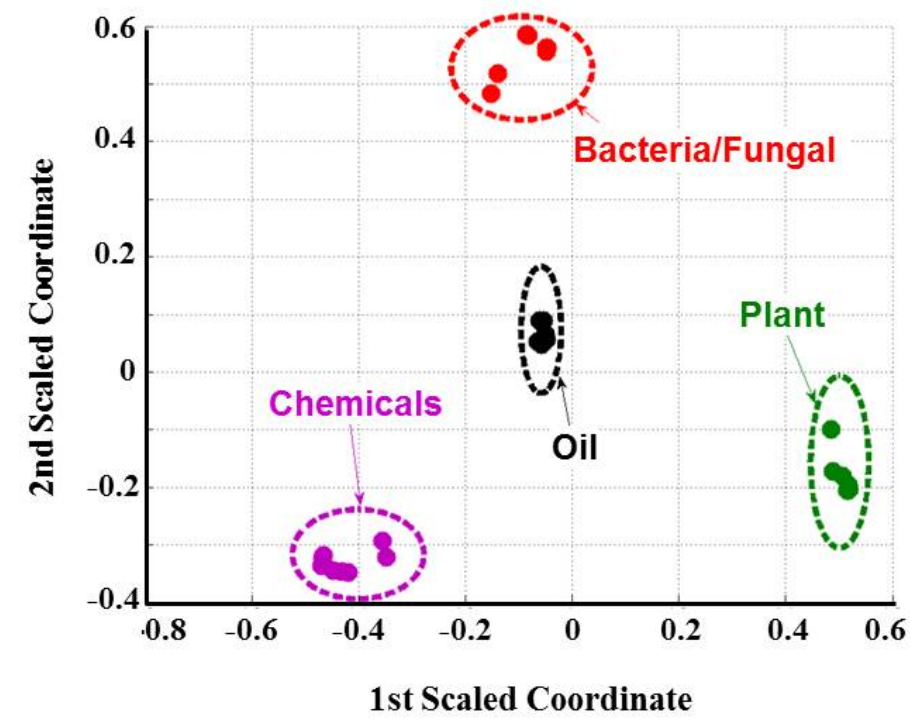

Figure 8. Plot of the measured samples in scaled coordinates. The scaled coordinates are the eigenvectors of the proximity matrix. The data are clustered into four groups: chemical, biological, oil, and plant material.

The prediction power of classification is displayed as averaged prediction confidence level for "out-of-bag" (OOB) samples (an unbiased estimator of the true ensemble error) and for complete training dataset (biased estimator) in Table 1. Both results present a high confidence above $90 \%$ of the proposed recognition system for all classes.

\begin{tabular}{|l|l|l|l|l|}
\hline Estimator & Chemicals & Bacteria & Plants & Oils \\
\hline Unbiased & 0.94 & 0.92 & 0.98 & 0.96 \\
\hline Biased & 0.97 & 0.95 & 0.98 & 0.95
\end{tabular}

Table 1: Average prediction confidence levels for unbiased and biased estimators. 


\section{CONCLUSIONS}

In this work, a wide variety of chemical and biological materials have been investigated for their fluorescence properties by using the laser induced fluorescence (LIF) technique. For this purpose, the substances were excited on a free transmission range, either as a liquid or as an aerosol, by laser light with two different wavelengths (280 and $355 \mathrm{~nm})$, while the returning fluorescence signals and fluorescence lifetimes were detected. Subsequently, the outputs were analyzed with dedicated software for spectral pattern recognition. The direct comparison of all results leads to a first classification of the various compounds. The classification of the measured samples is in the range of 92-98 \%. Thus, the laser induced fluorescence is a promising standoff measurement method for the discrimination of different materials and it can be used in combination with other standoff techniques for the characterization of hazardous materials.

As an outlook, we will enlarge the substance list, on the one hand, to consolidate the classification and, on the other hand, to define sub-classes within the classes, such as the differentiation between various bacterial strains.

\section{ACKNOWLEDGEMENTS}

Special thanks to Prof. Dr. Dirk M. Guldi and his working group from the Department of Physical Chemistry I, Friedrich-Alexander University Erlangen-Nuremberg. 


\section{REFERENCES}

[1] Handke, J., Duschek, F. and Pargmann, C., "Gefahrenerkennung mit Lichtgeschwindigkeit," Laser Technik Journal 7(4), 43-46 (2010).

[2] Wallin, S., Pettersson, A., Östmark, H. and Hobro, A., "Laser-based standoff detection of explosives: a critical review," Analytical and Bioanalytical Chemistry, Review (2009).

[3] Meyer, O., Jacquelard, C., Melkonian, J. M., Chardard, P., Lanson, P. and Petitgas, D., "Stand-off biological detection by LIF (laser induced fluorescence) LIDAR," Optronics in Defence and Security, 4th International Symposium

[4] Sivaprakasam, V., Lin, H. B., Huston, A. L. and Eversole, J. D., "Spectral characterization of biological aerosol particles using two-wavelength excited laser-induced fluorescence and elastic scattering measurements," Optics Express 19, 6191-6208 (2011).

[5] Mierczyk, Z., Kopczynski, K., Zygmunt, M., Wojtanowski, J., Mlynczak, J., Gawlikowski, A., Mlodzianko, A., Piotrowski, W., Gietka, A., Knysak, P., Drozd, T., Muzal, M., Kaszczuk, M., Ostrowski, R. and Jakubaszek, M., "Fluorescence/depolarization lidar for mid-range stand-off detection of biological agents," Laser Radar Technology and Applications XVI(8037), Proc. of SPIE (2011).

[6] Bruce, L. M., Koger, C. H., Li, J., "Dimensionality reduction of hyperspectral data using discrete wavelet transform feature extraction," IEEE T. Geoscience and Remote Sensing 40(10), 2331-2338 (2002).

[7] Mojaradi, B., Moghaddam, H. A., Zoej, M. J. V., Duin, R. P. W., "Dimensionality Reduction of Hyperspectral Data via Spectral Feature Extraction," IEEE T. Geoscience and Remote Sensing 47(7-2), 2091-2105 (2009).

[8] Wu, K.-L., Hsieh, P.-F., "Empirical mode decomposition for dimensionality reduction of hyperspectral data," IGARSS, IEEE, 1241-1244 (2005).

[9] Sobolev, I., Babichenko, S., "Analysis of the performances of hyperspectral lidar for water pollution diagnostics," EARSeL eProceedings 12(2), 113-123 (2013).

[10] Breiman, L., "Bagging Predictors," Machine Learning 24(2), 123-140 (1996).

[11] Breiman, L., "Random forests," Machine Learning 45, 5-32 (2001). 